\title{
NEW EVIDENCE SUPPORTING CLUSTER MEMBERSHIP FOR THE KEYSTONE CALIBRATOR DELTA CEPHEI
}

\author{
D. Majaess ${ }^{1}$, D. Turner ${ }^{1}$, ANd W. Gieren ${ }^{2}$ \\ ${ }^{1}$ Department of Astronomy and Physics, Saint Mary's University, Halifax, NS, Canada; dmajaess@cygnus.smu.ca \\ ${ }^{2}$ Departamento de Astronomía, Universidad de Concepción, Concepción, Chile \\ Received 2011 November 14; accepted 2012 January 3; published 2012 February 24
}

\begin{abstract}
New and existing $U B V J H K_{s}$, spectroscopic, NOMAD, Hubble Space Telescope, and revised Hipparcos observations are employed to determine properties for $\delta$ Cep and its host star cluster. The multi-faceted approach ensured that uncertainties were mitigated $(\sigma / d \sim 2 \%)$. The following fundamental parameters were inferred for $\delta$ Cep: $E(B-V)=0.073 \pm 0.018(\sigma), \log \tau=7.9 \pm 0.1$, and $d=272 \pm 3\left(\sigma_{\bar{x}}\right) \pm 5(\sigma)$ pc. The cluster exhibits a turnoff near B6 $\left(M_{*} / M_{\odot} \sim 5\right)$, and the brightest host cluster members are the supergiants $\zeta$ Cep (K1.5Ib) and $\delta$ Cep. To within the uncertainties, the two stars share common astrometric parameters $\left(\pi, \mu_{\alpha}, \mu_{\delta}, \mathrm{RV} \sim-17 \mathrm{~km} \mathrm{~s}^{-1}\right)$ and are tied to bluer members via the evolutionary track implied by the cluster's $U B V J H K_{s}$ color-color and color-magnitude diagrams. The cluster's existence is bolstered by the absence of an early-type sequence in color-magnitude diagrams for comparison fields. NOMAD data provided a means to identify potential cluster members $(n \sim 30)$ and double the existing sample. That number could increase with forthcoming precise proper motions (DASCH) for fainter mainsequence stars associated with classical Cepheids (e.g., $\delta$ Cep), which may invariably foster efforts to strengthen the Galactic Cepheid calibration and reduce uncertainties tied to $H_{0}$.
\end{abstract}

Key words: Hertzsprung-Russell and C-M diagrams - open clusters and associations: general - stars: distances stars: variables: Cepheids

Online-only material: color figures

\section{INTRODUCTION}

Cepheid variables are utilized to establish extragalactic distances and constrain cosmological models (Macri \& Riess 2009; Shappee \& Stanek 2011; Riess et al. 2011). However, the reliability of the derived parameters is invariably tied to the Cepheid calibration. Freedman et al. (2001) noted that ambiguities related to the zero point of the calibration account for a sizable fraction of the total uncertainty associated with $H_{0}$. The uncertainty hinders efforts to constrain dark energy, since the parameter is acutely dependent on an accurate Hubble constant $\left(\sigma_{\mathrm{w}} \sim 2 \sigma_{\mathrm{H}_{0}}\right.$; Macri \& Riess 2009). The next generation follow-up to the Hubble Space Telescope (HST) key project to measure $H_{0}$ (the Hubble Carnegie project; Freedman \& Madore 2010) aims to mitigate that problem by relying on LMC and Galactic calibrators (Benedict et al. 2002, 2007; Turner 2010; Storm et al. 2011). Consequently, bolstering the Galactic calibration should support efforts by the Hubble Carnegie, Araucaria, and $\mathrm{SH}_{0} \mathrm{ES}$ projects to determine $H_{0}$ to within $2 \%-4 \%$ (Gieren et al. 2005; Riess et al. 2011).

In this study, $U B V J H K_{s}$, spectroscopic, NOMAD, $H S T$, and revised Hipparcos (HIP) observations for stars physically associated with $\delta$ Cep are employed to constrain its fundamental parameters: age $(\log \tau)$, color excess $\left(E_{B-V}\right)$, distance, progenitor mass, and absolute Wesenheit magnitude $\left(W_{V I_{c}, 0}\right)$.

\section{ANALYSIS}

\subsection{Revised Hipparcos Observations for Cep OB6}

In their comprehensive study, de Zeeuw et al. (1999) discovered that $\delta$ Cep was a member of a group subsequently denoted as Cep OB6 (see also Hoogerwerf et al. 1997). Twenty stars identified by de Zeeuw et al. (1999) as Cep OB6 members are highlighted in Table 1. Stars exhibiting spectral types inconsistent with cluster membership based on their $U B V J H K_{s}$ color-color and color-magnitude positions were flagged as nonmembers. For example, HIP 110459 was previously assigned a membership probability of $100 \%$ (Table 1), yet the star exhibits $J H K_{s}$ photometry and a late-type temperature class (K5; Skiff 2010) indicative of a field red clump giant (Figure 2). $U B V J H K_{s}$ photometry was obtained from Mermilliod (1991) and Two Micron All Sky Survey (2MASS; Cutri et al. 2003). Spectral types were assigned to stars featured in the Catalogue of Stellar Spectral Classifications (Skiff 2010).

Revised HIP parallaxes (van Leeuwen 2007) were tabulated for 15 stars in Table 1 which were identified as probable cluster members. The revised HIP parallaxes exhibit a $\sim 30 \%$ reduction in uncertainties relative to existing data (Perryman \& ESA 1997), and the parallaxes for $\delta$ Cep and HD 213307 $(r \sim 0.7)$ were increased from $\pi=3.32 \pm 0.58: 3.43 \pm 0.64$ to $\pi=3.77 \pm 0.16: 3.69 \pm 0.46$ mas. A mean distance for the revised cluster sample outlined in Table 1 is $d=$ $271 \pm 11\left(\sigma_{\bar{x}}\right) \pm 42(\sigma)$ pc (see also de Zeeuw et al. 1999, and their Appendix B).

\subsection{Reddening}

$U B V J H K_{s}$ color-color analyses permit an assessment of the sample's extinction properties. $U B V$ data are particularly efficient at identifying early-type stars owing to the $U$-band's sensitivity to the Balmer decrement (e.g., Turner 1989; Carraro et al. 2006).

Cluster members featuring $U B V$ photometry (Mermilliod 1991) are offset from the intrinsic $U B V$ color-color relation by $E(B-V)=0.077 \pm 0.016(\sigma)$ (Table 1 and Figure 1 ). The findings support the field reddening determined for $\delta$ Cep by Benedict et al. (2002) and are consistent with those established from spectroscopic and $J H K_{s}$ observations. However, 
Table 1

Cep OB6 Member List (Z99)

\begin{tabular}{|c|c|c|c|c|c|c|}
\hline HIP ID & $\begin{array}{l}\mathrm{HIP} \pi^{\mathrm{a}} \\
\text { (mas) }\end{array}$ & $\begin{array}{l}\mathrm{V} 07 \pi^{\mathrm{a}} \\
\text { (mas) }\end{array}$ & $\begin{array}{c}\mu_{\alpha}, \mu_{\delta}^{\mathrm{b}} \\
\left(\mathrm{mas} \mathrm{yr}^{-1}\right)\end{array}$ & m.p. $(Z 99)^{\mathrm{c}}$ & m.p. ${ }^{\text {d }}$ & $E(B-V)^{\mathrm{e}}$ \\
\hline 109426 & $3.8 \pm 0.7$ & $3.6 \pm 0.5$ & $16.6 \pm 0.6,4.1 \pm 0.5$ & 94 & $\mathrm{~m}$ & $\cdots$ \\
\hline 109492 & $4.5 \pm 0.5$ & $3.9 \pm 0.1$ & $13.3 \pm 0.4,4.4 \pm 0.3$ & 97 & $\mathrm{~m}$ & $\cdots$ \\
\hline 110266 & $3.9 \pm 0.6$ & $4.4 \pm 0.3$ & $19.0 \pm 0.5,5.1 \pm 0.5$ & 96 & $\mathrm{~m}$ & 0.070 \\
\hline 110275 & $4.0 \pm 1.0$ & $4.0 \pm 0.9$ & $14.7 \pm 1.0,5.7 \pm 0.9$ & 89 & $\mathrm{~m}$ & $\ldots$ \\
\hline 110356 & $3.4 \pm 0.7$ & $2.9 \pm 0.5$ & $11.7 \pm 0.6,3.2 \pm 0.6$ & 100 & $\mathrm{~m}$ & 0.085 \\
\hline 110497 & $3.8 \pm 0.6$ & $3.2 \pm 0.4$ & $17.4 \pm 0.5,4.8 \pm 0.5$ & 98 & $\mathrm{~m}$ & 0.060 \\
\hline 110648 & $3.9 \pm 1.0$ & $3.3 \pm 0.9$ & $16.2 \pm 1.0,6.2 \pm 0.8$ & 84 & $\mathrm{~m}$ & $\cdots$ \\
\hline 110807 & $4.0 \pm 0.6$ & $3.5 \pm 0.4$ & $16.1 \pm 0.5,5.4 \pm 0.4$ & 92 & $\mathrm{~m}$ & 0.060 \\
\hline 110925 & $4.3 \pm 0.9$ & $5.1 \pm 0.8$ & $21.3 \pm 1.1,4.8 \pm 0.8$ & 86 & $\mathrm{~m}$ & $0.060^{f}$ \\
\hline 110988 & $3.4 \pm 0.6$ & $3.7 \pm 0.5$ & $16.4 \pm 0.7,4.7 \pm 0.7$ & 100 & $\mathrm{~m}$ & $0.085^{\mathrm{f}}$ \\
\hline 111060 & $5.0 \pm 0.8$ & $5.3 \pm 0.7$ & $17.2 \pm 0.7,4.5 \pm 0.7$ & 100 & $\mathrm{~m}$ & $\cdots$ \\
\hline 112141 & $3.2 \pm 0.8$ & $3.4 \pm 0.6$ & $14.6 \pm 0.7,2.1 \pm 0.6$ & 89 & $\mathrm{~m}$ & $\cdots$ \\
\hline 113255 & $4.3 \pm 0.7$ & $4.5 \pm 0.5$ & $19.8 \pm 0.6,3.0 \pm 0.6$ & 99 & $\mathrm{~m}$ & 0.095 \\
\hline 113316 & $3.2 \pm 0.7$ & $3.6 \pm 0.4$ & $14.1 \pm 0.7,3.2 \pm 0.6$ & 99 & $\mathrm{~m}$ & 0.100 \\
\hline$\delta$ Cep & $3.3 \pm 0.6$ & $3.8 \pm 0.2$ & $16.4 \pm 0.6,3.5 \pm 0.6$ & 89 & $\mathrm{~m}$ & $\cdots$ \\
\hline 110459 & $4.1 \pm 0.9$ & $4.5 \pm 0.7$ & $16.2 \pm 0.9,5.2 \pm 0.7$ & 100 & $\mathrm{~nm}$ & $\cdots$ \\
\hline 111069 & $3.2 \pm 0.9$ & $3.1 \pm 0.8$ & $15.1 \pm 0.8,6.9 \pm 0.7$ & 79 & $\mathrm{~nm}$ & $\cdots$ \\
\hline 112473 & $3.6 \pm 0.8$ & $5.0 \pm 0.7$ & $13.9 \pm 0.8,2.8 \pm 0.8$ & 97 & $\mathrm{~nm}$ & $\cdots$ \\
\hline 112998 & $2.5 \pm 0.6$ & $2.6 \pm 0.3$ & $12.7 \pm 0.6,2.2 \pm 0.5$ & 98 & $\mathrm{~nm}$ & $\cdots$ \\
\hline 113993 & $3.8 \pm 0.7$ & $3.7 \pm 0.4$ & $14.3 \pm 0.6,3.9 \pm 0.5$ & 79 & $\mathrm{~nm}$ & $\cdots$ \\
\hline
\end{tabular}

Notes.

a Parallaxes from Perryman et al. (1997, HIP) and van Leeuwen (2007, V07).

b Proper-motion data from NOMAD (Zacharias et al. 2004).

c Membership probability assigned by de Zeeuw et al. (1999, Z99).

${ }^{\mathrm{d}}$ Membership inferred from $U B V J H K_{s}$ and spectroscopic observations (Figures 1 and 2).

e Reddenings derived from the $U B V$ color-color analysis (Figure 1).

${ }^{\mathrm{f}}$ Stars in close proximity to $\delta$ Cep.

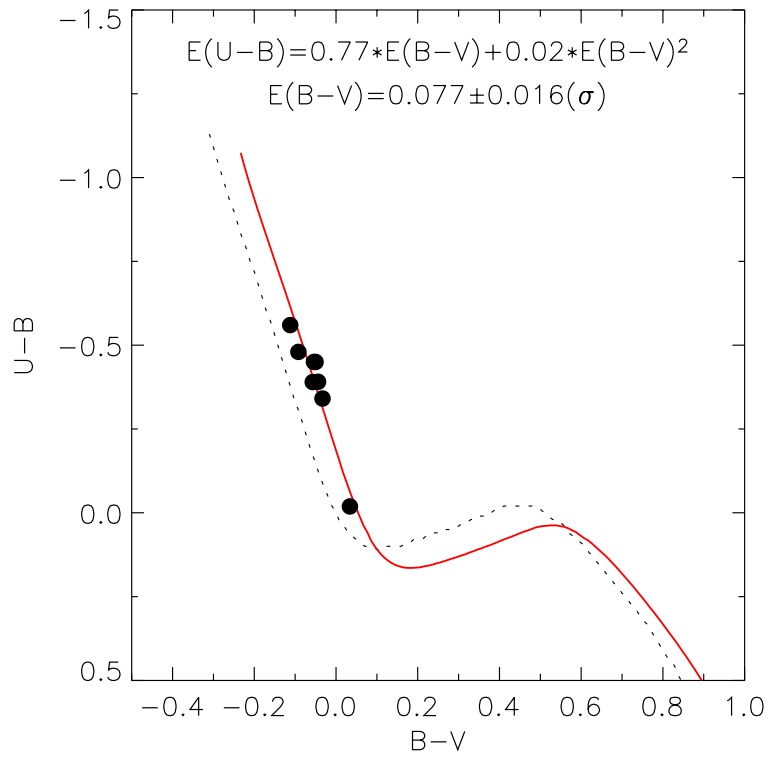

Figure 1. Color-color diagram for stars in Table 1 which are associated with $\delta$ Cep and possess $U B V$ photometry. The sample is offset from the intrinsic relation (dotted line) by $E(B-V)=0.077 \pm 0.016(\sigma)$ (solid line). The result confirms the reddening established for $\delta$ Cep by Benedict et al. (2002). The intrinsic relation and reddening law for the region were adopted from Turner (1976, 1989).

(A color version of this figure is available in the online journal.)

uncertainties associated with the latter hamper a precise assessment. The minimal spread, $E(B-V) \sim 0.06-0.10$ (Table 1), may be indicative of marginal differential reddening, rotation, binarity, or photometric uncertainties.

\subsection{Age}

$U B V J H K_{s}$ color-color analyses imply a cluster turnoff near B5-B7 $\left(M_{*} / M_{\odot} \sim 5\right)$, according to intrinsic relations (Padova models; Straižys \& Lazauskaitė 2009; Turner 1976, 1989, 2011). A cluster age of $\log \tau=7.9 \pm 0.1$ was inferred from the turnoff determined (Figure 1 and Table 1), and since the corresponding Padova evolutionary track (Figure 2) matches bluer and redder evolved members ( $\delta$ and $\zeta$ Cep). The result agrees with the Cepheid's predicted age (Turner 1996; Bono et al. 2005). The temporal match is pertinent evidence in support of cluster membership for $\delta$ Cep.

\subsection{Cluster Distance}

A precise distance may be established since two of four principal parameters associated with isochrone fitting were constrained by the $U B V J H K_{s}$ color-color and spectroscopic analyses, namely, the reddening and age (spectral type at the turnoff). $\delta$ Cep exhibits solar abundances, and hence the remaining parameter is the shift required in magnitude space to overlay the intrinsic relation upon the data. The resulting distance is $d=277 \pm 15 \mathrm{pc}$ (Figure 2). The zero point is tied to seven benchmark open clusters $(d<250 \mathrm{pc})$ which exhibit matching $J H K_{s}$ and revised Hipparcos distances (the Hyades, $\alpha$ Per, Praesepe, Coma Ber, IC 2391, IC 2609, and NGC 2451; van Leeuwen 2009; Majaess et al. 2011a). A redetermination of the HST parallax for the Hyades supports that scale (McArthur et al. 2011). Isochrones, models, and the distance scale should be anchored (and evaluated) using clusters where consensus exists, rather than the discrepant case (i.e., the Pleiades). A ratio of total to selective extinction $R_{J}$ was adopted from Majaess 


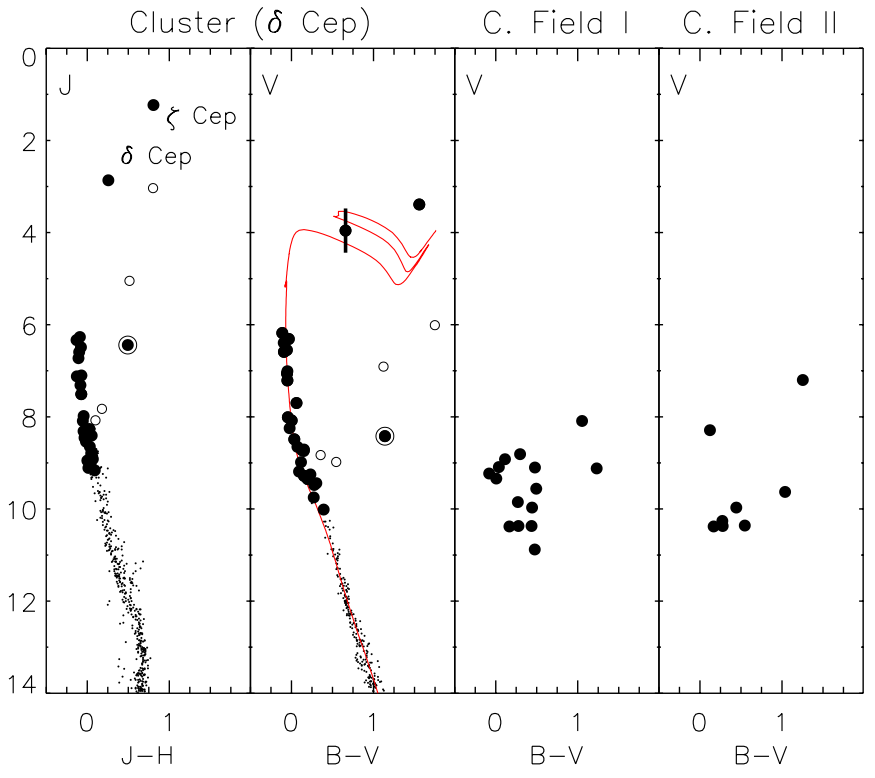

Figure 2. Left to right: $J H$ and $B V$ color-magnitude diagrams for the Table 1 and NOMAD samples (panels 1 and 2), and comparison fields (panels 3 and 4). Small dots denote calibration stars from Majaess et al. (2011a), which were employed to tie the cluster distance to a geometrically anchored scale (van Leeuwen 2009; Majaess et al. 2011a). Large dots characterize stars ex-

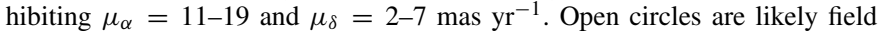
stars (Table 1). HIP 110459 (circled dot) was previously identified as a cluster member, yet $B V J H K_{s}$ photometry implies the object is a field star. Panel 2: a Padova $\log \tau=7.9$ isochrone was applied. The brightest cluster members are likely the supergiants $\zeta$ Cep $(\mathrm{K} 1.5 \mathrm{Ib})$ and $\delta \mathrm{Cep}$ (amplitude variation indicated). An early-type cluster sequence is absent from the comparison fields (HIP data for the cluster $\left.\mu_{\alpha} / \mu_{\delta}\right)$.

(A color version of this figure is available in the online journal.)

et al. (2011b; see also Bonatto et al. 2004), whereas a value for $R_{V}$ was adopted from Turner (1976). An advantage of employing $J H K_{S}$ observations is that the cluster reddening is negligible in that part of the spectrum $\left(E_{J-H} \sim 0.3 \times E_{B-V}\right.$, Majaess et al. 2008; Bonatto et al. 2004, and references therein), which consequently mitigates the impact of uncertainties in $R_{\lambda}$ $\left(J_{0}=J-E_{J-H} \times R_{J}\right)$.

The distance derived from the cluster color-magnitude diagrams (Figure 2) is tied to additional potential cluster members identified using NOMAD (Zacharias et al. 2004). That database was queried for stars exhibiting $\mu_{\alpha}=11-19$ and $\mu_{\delta}=2-7$ mas $\mathrm{yr}^{-1}$ (Table 1). Stars fainter than $J \sim 9.2$ were culled from the resulting sample to reduce field contamination. Proper motions may be less reliable for such stars, and spectroscopic and $U B V$ observations are typically unavailable. Stars redder than $J-H \sim 0.4$ were likewise removed to mitigate contamination from field red clump giants. In addition, stars featuring anomalous positions in the multiband color-color and color-magnitude diagrams were removed. The remaining stars double the number of existing potential cluster members and are highlighted in Table 2 . The stars in Tables 1 and 2 are potential members pending further evidence. The NOMAD proper motions are consistent with estimates from the PPMXL catalog (Table 2). UBVJHK photometry was sought from Mermilliod (1991), 2MASS (Cutri et al. 2003), and pertinent resources. For example, new observations acquired from the Bright Star Monitor, which is part of the AAVSO's robotic telescope network, provided $B V$ photometry for HD 239949: $V=10.013 \pm 0.031$ and $B-V=0.392 \pm 0.048$. The cluster reddening was redetermined $\left(E_{B-V}=0.073 \pm 0.018(\sigma)\right)$ after including four
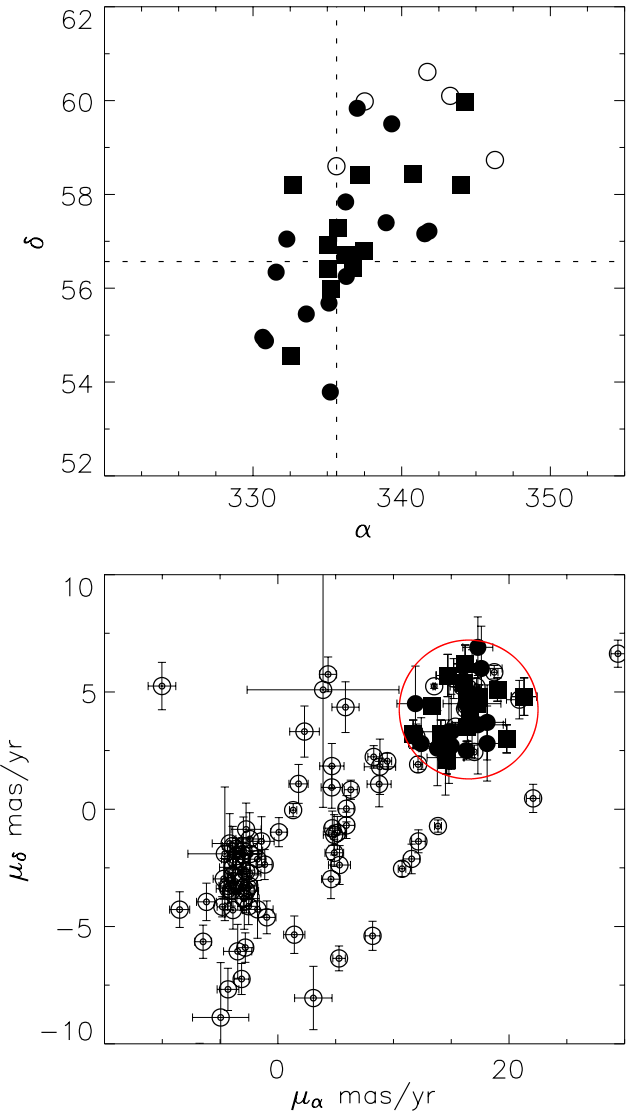

Figure 3. Top: J2000 R.A./decl. positions for probable (squares) and nonmembers (open circles) highlighted in Table 1, and new potential members (filled circles) outlined in Table 2. Bottom: dotted open circles represent all HIP stars near the (approximate) cluster center.

(A color version of this figure is available in the online journal.)

earlier type stars highlighted in Table 2 which possess $U B V$ photometry.

Cluster members appear to aggregate near J2000 coordinates of $22^{\mathrm{h}} 22^{\mathrm{m}} .5+56^{\circ} 34^{\prime}$ (Figure 3 ). $\delta$ Cep lies at the periphery of the density enhancement, and within the confines of the cluster since the corona extends further (Kholopov 1969; Turner 1985). The Cepheid is $r \sim 2^{\circ}$ from the aforementioned coordinates, which is equivalent to a linear projected separation of $\sim 9 \mathrm{pc}$. The revised HIP and HST parallaxes for $\delta$ Cep and HD 213307 ( $r \sim 0$.'7 from $\delta \mathrm{Cep}$ ), in tandem with their apparent positions, suggest that the distance to the cluster center and Cepheid is analogous to within the uncertainties.

\subsection{Mean Distance to $\delta$ Cep}

The mean HIP parallax for the cluster (Section 2.1) agrees with the HIP parallax for $\delta \mathrm{Cep}(\pi=3.77 \pm 0.16$ mas), the $H S T$ parallax for $\delta \operatorname{Cep}(\pi=3.66 \pm 0.15$ mas; Benedict et al. 2002), and the distance inferred for the host cluster from $U B V J H K_{s}$ and spectroscopic observations (Figures 1 and 2). Assigning equal weight to each method, the mean of the four distances for $\delta$ Cep is: $d=272 \pm 3\left(\sigma_{\bar{x}}\right) \pm 5(\sigma)$ pc. That result agrees with the Storm et al. (2011) determination from the infrared surface brightness technique (IRSB; Fouque \& Gieren 1997). The associated standard error and deviation provide a realistic estimate for the systematic uncertainty, which is often difficult to characterize. 
Table 2

Additional Potential Cluster Members

\begin{tabular}{|c|c|c|c|c|c|c|c|c|}
\hline ID & $V$ & $B-V$ & $U-B$ & $J$ & $H$ & $K_{s}$ & $\begin{array}{c}\mu_{\alpha}, \mu_{\delta}^{\mathrm{a}} \\
\left(\mathrm{mas} \mathrm{yr}^{-1}\right)\end{array}$ & $\begin{array}{c}\mu_{\alpha}, \mu_{\delta}^{\mathrm{b}} \\
\left(\mathrm{mas}_{\mathrm{yr}}^{-1}\right)\end{array}$ \\
\hline HD $210071^{c}$ & 6.39 & -0.10 & -0.45 & 6.49 & 6.57 & 6.58 & $16.3 \pm 0.4,2.5 \pm 0.4$ & $16.3 \pm 0.4,2.4 \pm 0.4$ \\
\hline HD $209636^{c}$ & 7.01 & -0.05 & -0.23 & 7.10 & 7.17 & 7.16 & $15.0 \pm 0.5,2.7 \pm 0.5$ & $15.0 \pm 0.5,2.6 \pm 0.5$ \\
\hline HD 214259 & 8.72 & 0.15 & 0.10 & 8.27 & 8.24 & 8.24 & $18.1 \pm 1.6,3.7 \pm 1.6$ & $18.4 \pm 1.3,4.3 \pm 1.3$ \\
\hline HD 214512 & 8.74 & 0.15 & $\ldots$ & 8.30 & 8.29 & 8.29 & $17.6 \pm 1.8,6.0 \pm 1.8$ & $-21.5 \pm 1.6,53.1 \pm 1.6$ \\
\hline HD 212093 & 8.25 & -0.02 & -0.29 & 8.31 & 8.36 & 8.35 & $15.0 \pm 1.6,3.3 \pm 1.6$ & $14.1 \pm 1.2,2.5 \pm 1.2$ \\
\hline HD 210480 & 8.71 & 0.15 & 0.08 & 8.41 & 8.35 & 8.31 & $14.5 \pm 1.6,2.2 \pm 1.6$ & $14.9 \pm 1.3,2.3 \pm 1.3$ \\
\hline HD 211226 & 8.65 & 0.07 & 0.03 & 8.45 & 8.49 & 8.47 & $17.3 \pm 1.3,6.9 \pm 1.3$ & $15.4 \pm 1.2,6.7 \pm 1.2$ \\
\hline HD 215879 & 8.98 & 0.12 & $\ldots$ & 8.65 & 8.62 & 8.59 & $13.8 \pm 1.6,2.6 \pm 1.6$ & $13.4 \pm 1.3,4.4 \pm 1.2$ \\
\hline HD 212711 & 9.25 & 0.23 & $\ldots$ & 8.78 & 8.72 & 8.66 & $17.3 \pm 2.2,3.6 \pm 2.1$ & $16.7 \pm 1.9,5.2 \pm 1.9$ \\
\hline HD 240052 & 9.44 & 0.30 & $\cdots$ & 8.79 & 8.74 & 8.71 & $18.1 \pm 1.6,2.8 \pm 1.6$ & $17.3 \pm 1.3,4.1 \pm 1.2$ \\
\hline HD 212137 & 9.19 & 0.09 & $\ldots$ & 8.95 & 8.95 & 8.94 & $12.4 \pm 1.1,2.8 \pm 1.1$ & $12.9 \pm 1.2,1.8 \pm 1.2$ \\
\hline $\mathrm{BD}+54^{\circ} 2675$ & 9.48 & 0.28 & $\cdots$ & 9.04 & 9.01 & 8.94 & $11.9 \pm 1.6,4.5 \pm 1.6$ & $12.2 \pm 1.8,7.5 \pm 1.7$ \\
\hline HD 239949 & 10.01 & 0.39 & $\cdots$ & 9.11 & 9.10 & 9.06 & $16.8 \pm 2.5,4.5 \pm 2.3$ & $5.4 \pm 1.6,-0.8 \pm 1.5$ \\
\hline $\mathrm{BD}+59^{\circ} 2523$ & 9.75 & 0.27 & $\ldots$ & 9.16 & 9.07 & 9.11 & $14.8 \pm 2.3,3.3 \pm 2.2$ & $15.3 \pm 1.6,4.2 \pm 1.6$ \\
\hline
\end{tabular}

Notes.

a NOMAD proper motions (Zacharias et al. 2004).

b The PPMXL catalog (Roeser et al. 2010).

${ }^{\mathrm{c}} \pi=5.06 \pm 0.33: 5.54 \pm 0.39$ mas (van Leeuwen 2007).

The resulting $V I_{c}$ Wesenheit magnitude for $\delta$ Cep is $W_{V I_{c}, 0}=$ $-5.12\left(R_{V I_{c}}=2.55\right)$. That is consistent with results established for classical Cepheids displaying similar pulsation periods: CV Mon, V Cen, Y Sgr, and CS Vel (Benedict et al. 2007; Turner 2010; Majaess et al. 2011b).

\section{CONCLUSION AND FUTURE RESEARCH}

The evidence presented bolsters the assertion by de Zeeuw et al. (1999) that $\delta$ Cep is a constituent of an intermediate-age cluster. The brightest cluster member is the K1.5Ib supergiant $\zeta$ Cep. $\delta$ and $\zeta$ Cep share similar HIP parallaxes $(\pi=$ $3.77 \pm 0.16: 3.90 \pm 0.10$ mas), proper motions, radial velocities $\left(\mathrm{RV} \sim-17 \mathrm{~km} \mathrm{~s}^{-1}\right)$, and evolutionary histories (Figure 2). In tandem with the aforementioned evidence, the cluster's existence is supported by the absence of early-type stars from the comparison fields (Figure 2). NOMAD data were employed to identify additional potential cluster members (Tables 1 and 2).

The Cepheid exhibits parameters of: $E(B-V)=0.073 \pm$ $0.018(\sigma), \log \tau=7.9 \pm 0.1$, and $d=272 \pm 3\left(\sigma_{\bar{x}}\right) \pm 5(\sigma) \mathrm{pc}$ (Table 1, and Figures 1 and 2). The results are tied in part to spectroscopic and $U B V J H K_{s}$ observations, and may be adopted to refine classical Cepheid period-color, period-age, periodmass, period-luminosity, and period-Wesenheit relations (e.g., Turner 2010).

Potential future research entails establishing precise proper motions for fainter stars near Cepheids using photographic plates stored at the CfA (Grindlay 2007; DASCH), ${ }^{3}$ thereby extending the astrometric coverage provided by HIP. The plates stored at the CFA offer unmatched multi-epoch observations over a $\sim 100$ year temporal baseline. A concurrent venture pertains to employing new VVV $J H K_{s}$ observations for young clusters in Galactic spiral arms to calibrate adjacent long-period classical Cepheids (Minniti et al. 2010; Moni Bidin et al. 2011; Majaess et al. 2011b). The aforementioned initiatives, in harmony with the analysis presented here, shall complement a suite of diverse efforts aimed at reducing uncertainties associated with

\footnotetext{
3 Digital Access to a Sky Century @ Harvard (DASCH),
} http://hea-www.harvard.edu/DASCH/.
$H_{0}$ in order to constrain cosmological models (e.g., Benedict et al. 2002, 2007; Feast 2008; Gerke et al. 2011; Ngeow 2011).

The agreement between the distances inferred for $\delta$ Cep from cluster membership and the IRSB technique suggests that the systematic uncertainties have been marginalized. An analysis of $n=20$ cluster Cepheids featuring revised IRSB distances (Storm et al. 2011) yields a mean fractional difference of $-3 \% \pm 3 \%$. That result is reassuring, and subsequent research on the discrepant Cepheid calibrators (e.g., S. Vul \& S. U. Cas, in preparation) may reduce the remaining offset. Further research is likewise required on the candidate cluster members associated with $\delta$ Cep (Tables 1 and 2).

D.M. is grateful to the following individuals and consortia whose efforts lie at the foundation of the research: F. van Leeuwen and M. Perryman (HIP), F. Benedict (HST), P. de Zeeuw, R. Hoogerwerf, J-C. Mermilliod, B. Skiff, NOMAD, 2MASS, AAVSO (T. Krajci, A. Henden, and M. Simonsen), and staff at the CDS, arXiv, and NASA ADS. W.G. gratefully acknowledges financial support for this work from the Chilean Center for Astrophysics FONDAP 15010003, and from the BASAL Centro de Astrofisica y Tecnologias Afines (CATA) PFB-06/2007.

\section{REFERENCES}

Benedict, G. F., McArthur, B. E., Feast, M. W., et al. 2007, AJ, 133, 1810 Benedict, G. F., McArthur, B. E., Fredrick, L. W., et al. 2002, AJ, 124, 1695 Bonatto, C., Bica, E., \& Girardi, L. 2004, A\&A, 415, 571 Bono, G., Marconi, M., Cassisi, S., et al. 2005, ApJ, 621, 966

Carraro, G., Chaboyer, B., \& Perencevich, J. 2006, MNRAS, 365, 867 Cutri, R. M., Skrutskie, M. F., van Dyk, S., et al. 2003, The IRSA 2MASS All-Sky Point Source Catalog, NASA/IPAC Infrared Science Archive de Zeeuw, P. T., Hoogerwerf, R., de Bruijne, J. H. J., Brown, A. G. A., \& Blaauw, A. 1999, AJ, 117,354

Feast, M. W. 2008, in First Middle East-Africa, Regional IAU Meeting, 2008 April 5-10, Cairo, Egypt, ed. A. W. Alsabti, A. A. Hady, \& V. Bothmer (Cairo: Univ. Cairo Press), 3 (arXiv:0806.3019)

Fouque, P., \& Gieren, W. P. 1997, A\&A, 320, 799

Freedman, W. L., \& Madore, B. F. 2010, ARA\&A, 48, 673

Freedman, W. L., Madore, B. F., Gibson, B. K., et al. 2001, ApJ, 553, 47

Gerke, J. R., Kochanek, C. S., Prieto, J. L., Stanek, K. Z., \& Macri, L. M. 2011, ApJ, 743, 176 
Gieren, W., Pietrzyński, G., Soszyński, I., et al. 2005, ApJ, 628, 695

Grindlay, J. E. 2007, in ASP Conf. Ser. 373, The Central Engine of Active Galactic Nuclei, ed. L. C. Ho \& J.-M. Wang (San Francisco, CA: ASP), 711

Hoogerwerf, R., de Bruijne, J. H. J., Brown, A. G. A., et al. 1997, in Proc. ESA Symp., Hipparcos-Venice'97, ed. M. A. C. Perryman, P. L. Bernacca, \& B. Battrick (ESA SP-402; Noordwijk: ESA), 571

Kholopov, P. N. 1969, SvA, 12, 625

Macri, L. M., \& Riess, A. G. 2009, AIP Conf. Ser. 1170, Stellar Pulsation: Challenges for Theory and Observation, ed. J. A. Guzik \& P. A. Bradley (Melville, NY: AIP), 23

Majaess, D. J., Turner, D. G., \& Lane, D. J. 2008, MNRAS, 390, 1539

Majaess, D. J., Turner, D. G., Lane, D. J., \& Krajci, T. 2011a, J. Am. Assoc. Var. Star Obs., 39, 219

Majaess, D., Turner, D., Moni Bidin, C., et al. 2011b, ApJ, 741, L27

McArthur, B. E., Benedict, G. F., Harrison, T. E., \& van Altena, W. 2011, AJ, 141,172

Mermilliod, J.-C. 1991, Homogeneous Means in the UBV System, VizieR Catalog

Minniti, D., Lucas, P. W., Emerson, J. P., et al. 2010, New Astron., 15, 433

Moni Bidin, C., Mauro, F., Geisler, D., et al. 2011, A\&A, 535, A33
Ngeow, C.-C. 2011, in Proc. 9th Pacific Rim Conference on Stellar Astrophysics (PRCSA2011), 2011 April, Lijiang, China (San Francisco, CA: ASP), arXiv:1111.2094

Perryman, M. A. C., ESA, \& FAST Consortium 1997, The Hipparcos and Tycho Catalogues (ESA Special Publication; Noordwijk: ESA), 1200

Riess, A. G., Macri, L., Casertano, S., et al. 2011, ApJ, 730, 119

Roeser, S., Demleitner, M., \& Schilbach, E. 2010, AJ, 139, 2440

Shappee, B. J., \& Stanek, K. Z. 2011, ApJ, 733, 124

Skiff, B. 2010, General Catalogue of Stellar Spectral Classifications, VizieR Catalog

Storm, J., Gieren, W., Fouqué, P., et al. 2011, A\&A, 534, A94

Straižys, V., \& Lazauskaitè, R. 2009, Baltic Astron., 18, 19

Turner, D. 1985, in IAU Colloq. 82, Cepheids: Theory and Observation, ed. B. F. Madore (New York: Cambridge Univ. Press), 209

Turner, D. G. 1976, AJ, 81, 1125

Turner, D. G. 1989, AJ, 98, 2300

Turner, D. G. 1996, J. R. Astron. Soc. Can., 90, 82

Turner, D. G. 2010, Ap\&SS, 326, 219

Turner, D. G. 2011, RevMexAA, 47, 127

van Leeuwen, F. 2007, A\&A, 474, 653

van Leeuwen, F. 2009, A\&A, 497, 209

Zacharias, N., Monet, D. G., Levine, S. E., et al. 2004, BAAS, 36, 1418 